Private practice, Warsaw, Poland

${ }^{4}$ II Department of Psychiatry, Medical University of Warsaw, Warsaw, Poland

\title{
Psychological well-being and diabetes- -related distress in states of type 2 diabetes in the first multi-national Diabetes Attitudes, Wishes and Needs (DAWN) Study
}

\section{ABSTRACT}

Purpose. To examine well-being and diabetes-related distress across several common states differentiated in the course of type 2 diabetes.

Material and methods. Random samples of adults with type 2 diabetes were obtained from multiple countries in the first DAWN (Diabetes Attitudes, Wishes, and Needs) Study ( $n=3432$ ). All data were obtained during structured interviews. Criteria for defining states of diabetes included time since diagnosis of diabetes, the timing and nature of anti-hyperglycaemic medication regimens, and the timing and number of complications.

Results. Duration of diabetes closely corresponded to a set of typical states based on the criteria. Using analysis of covariance to control for confounding factors, diabetes-related distress and psychological well-being were significantly $(p<0.05)$ worse for persons with diabetes with more complications and more intense medication regimens. Longer duration of insulin use was significantly associated with more

Address for correspondence: prof. dr hab. n. med. Andrzej Kokoszka II Klinika Psychiatryczna

Warszawski Uniwersytet Medyczny

ul. Kondratowicza 8, 03-242 Warszawa

Phone/fax: 00483265892

e-mail: andrzej.kokoszka@wum.edu.pl

Clinical Diabetology 2019, 8, 3, 167-175

DOI: $10.5603 /$ DK.2019.0012

Received: 04.02.2019

Accepted: 03.04.2019 diabetes-related distress. Worse distress and well-being were significantly associated with the accumulation of complications over time, but were more strongly associated with recently diagnosed complications than with more distally diagnosed complications.

Conclusions. Well-being and distress varied over states as defined by the nature and timing of diagnoses and medications. The observed patterns were more complex than a linear model of disease staging would suggest. (Clin Diabetol 2019; 8, 3: 167-175)

Key words: psychological well-being, diabetes, type 2 diabetes, diseases states, diagnosis, complications, treatment regimen

\section{Introduction}

It is well-recognized that type 2 diabetes is a progressive disease [1]. It has been proposed that diabetes can be conceptualized in terms of stages, but differentiation of its stages is less clear than in other life-long illness. Stages of overt type 2 diabetes have been identified based on the need for insulin, including not insulin requiring and requiring insulin for control [2, 3]. Similar staging was accepted by the Japan Diabetes Society [4]. Disease staging by Gonnella [5] included complications as a criterion. Because the course of type 2 diabetes may not follow consecutively all described states, our notion of "states" occurring over the course of diabetes de-emphasizes the requirement that there be a fixed order of progression across the states. The majority of cases can be classified into several states 
according to concrete criteria reflecting the progression of diabetes, including duration of diabetes diagnosis, therapeutic regimen - lifestyle intervention, oral antihyperglycemic medications (OAM), and injections and occurrence of complications.

What is less clear is how the progression of diabetes disease severity manifests itself in the psychological life of people with diabetes - how do the states in the progression of diabetes affect their psychological adjustment to having diabetes? The early work on this topic conceptualized the progression in terms of the predictable crises of diabetes [6], starting with the diagnosis of diabetes through changes in the intensity of medication regimens necessary to control blood glucose levels, and the onset of diabetic complications, hospitalization and the threat of imminent death. This work suggested that these events are associated with changes in psychological adjustment, with the implication that for each event there is a crisis period followed by a period of accommodation.

In this paper we seek to develop a multi-dimensional typology of the states in the course of type 2 diabetes and examine the impact of these states on two indicators of psychological adjustment in people with diabetes - psychological well-being and diabetesrelated distress.

Our typology is based on two criteria that define the different states. The first criterion is the type of event. We identify three such events:

- diagnosis of diabetes;

- major intensification of glucose lowering treatment (first oral medication, first injected medication);

- diagnosis of a diabetic complication.

The second criterion is the recency/latency of a criterion event (diagnosis or major treatment intensification). For the purpose of this paper we define a recent event as one that has occurred within the last year, while events having occurred more than a year ago are not recent.

There is evidence to support an association of psychological adjustment with each of the criteria noted above. Having type 2 diabetes is associated with higher levels of depression [7], and type 2 diabetes is associated with greater severity of depressive symptoms among those newly diagnosed than those previously diagnosed [8]. Among people with type 2 diabetes those treated with insulin have higher levels of depression than those not using insulin $[9,10]$, although insulin initiation may be associated with a short-term reduction in depression [11, 12]. Complications are associated with greater depression or diabetes-related distress [7, 13-16], with some evidence that depression rises at onset and resolves over time [17, 18].

Another formal property of our typology is the nature of progression across states: 1 ) Mechanical models define the progression as entirely predictable; the next state to be occupied is entirely determined by the current state, with no reversal in the progression, no skipping of states, etc. 2) Probabilistic models regard the progression as only partially predictable; state reversal and skipping may occur. Hybrid typologies, like the one proposed here, are a combination of the two models. Treatment intensification involves probabilistic progression; the exact progression is not predetermined, but given a current level of treatment intensity some next states are more likely than others. Complications involves a mechanist progression; a person with diabetes must have one complication before they have two complications, and for the most part complications do not reverse themselves (although they may be successfully treated). Recency/latency involves a mechanistic progression in that an event must have been recent before it becomes non-recent, and a non-recent event cannot become recent (although additional events may occur, thereby adding another recent event to existing events, e.g., new complications).

The criteria defined above were used to generate a set of nine states representing a typical progression of diabetes ("typical" in that actual progression of any given patient may not follow the progression hypothesized here). In addition to the logical requirements of mechanical progression, we make two probabilistic assumptions based on empirical considerations:

- for treatment intensification no medication precedes oral medication, and oral medication precedes insulin;

- for the relationship between treatment intensification and complications insulin initiation precedes complications.

Thus, the resulting typical diabetes states (in order from earliest to latest) are:

- new diagnosis of diabetes, no medication, no complications;

- old diagnosis of diabetes, no medication, no complications;

- old diagnosis of diabetes, oral medication, no complications;

- old diagnosis of diabetes, recent initiation of insulin, no complications;

- old diagnosis of diabetes, old initiation of insulin, no complications;

- old diagnosis of diabetes, old initiation of insulin, recent complication only; 
- old diagnosis of diabetes, old initiation of insulin, one old complication;

- old diagnosis of diabetes, old initiation of insulin, one old complication and one recent complication;

- old diagnosis of diabetes, old initiation of insulin, two old complications.

Additional states beyond the last one could be postulated but they would involve the replication of the pattern represented by the last several states onset of a third complication and its transition to an old complication, followed by a fourth cycle of complications, etc. For the purpose of simplification, and given the difficult of obtaining a sample large enough and with a high enough level of complications to be able to examine additional states, we limit ourselves to these nine states.

Using the proposed multi-dimensional typology of disease states, we assess:

- the impact of our criteria on the psychological adjustment of people with diabetes;

- the degree to which the order of the states corresponds to the actual duration of diabetes for the people with diabetes occupying those states (i.e., represent a progression);

- the degree to which these states of diabetes capture variation in the indicators of psychological adjustment.

\section{Material and methods}

\section{Study background}

The study design of the first Diabetes Attitudes, Wishes and Needs (DAWN) Study, described in detail elsewhere [19, 20], was a cross-sectional survey. In 2001 telephone or face-to-face interviews (in respondents' native language) were conducted in 13 countries representing 11 regions in Asia, Australia, Europe, and North America. There were three independent surveys of random samples of respondents: 5,426 adults who self-identified as having diabetes ( $\sim 500$ per region), 2,750 physicians ( $\sim 200$ primary care physicians and $\sim 50$ diabetes specialists per region), and 1,122 nurses ( $\sim 50$ diabetes specialists and 50 generalists per region).

The study was conducted according to the Joint Guidelines on Pharmaceutical Research Practice of the British Healthcare Business Intelligence Alliance and the Association of the British Pharmaceutical Industry. Verbal informed consent was obtained from all respondents and participation was voluntary. Ethical approval of the study protocol and use of these data was obtained from the institutional review board at Loyola University Maryland (the Human Subjects Research Committee).

\section{Study subjects}

Inclusion criteria were age 18-80 and self-identified as diagnosed with diabetes for at least six months. Exclusion criteria were severe physical or mental illness. The selection quota was for approximately equal numbers of people with self-reported type 1 and type 2 diabetes mellitus. This paper uses data from all people with diabetes who could be classified as having type 2 diabetes mellitus according to the following criteria: diagnosed at or after age 40, and not treated with insulin both at diagnosis and at the time of the survey.

\section{Measures}

\section{Respondent demographics}

In addition to country of residence, respondent demographic characteristics included sex, age, marital status (married or not), and residential urbanicity (rural, suburban, small urban, large urban).

\section{Diabetes states}

There were three main criteria for defining disease states:

- time since diagnosis of diabetes (up to one year vs. more than one year);

- glucose control medication regimen (none, oral only, insulin and oral, insulin only);

- pattern of complications (absence/presence of complications with onset of more/less than one year).

There were two secondary criteria: time since insulin initiation (up to one year vs. more than one year) and number of complications. The number of complications was a count of conditions reported as being under treatment from a list including 15 possibilities.

\section{Psychological outcomes}

Well-being was assessed by the WHO-5 measure [21] (alpha $=0.83$ ). It has adequate validity both as a screening tool for depression and as an outcome measure in clinical trials [20]. The raw score is calculated by totaling the figures of the five answers. The raw score ranges from 0 to 25,0 representing worst possible and 25 representing best possible quality of life. To obtain a percentage score ranging from 0 to 100, the raw score is multiplied by 4 . A percentage score of 0 represents worst possible, whereas a score of 100 represents best possible well-being. Diabetes-related distress was assessed with a multi-item scale developed for this study and using the following seven items (alpha $=0.79$ ): being stressed about diabetes, burned out from coping with diabetes, tired of complying with medications, afraid diabetes is getting worse, diabetes-related worry about family responsibilities, diabetes-related worry 
about financial future, worry about hypoglycemia. Response options (Fully disagree $=0$, Mainly disagree $=1$, Mainly agree $=2$, Fully agree $=3$ ) were multiplied by $100 / 3$ and the score was calculated as the mean of completed items. Scores could range from 0 to 100 .

\section{Statistical analysis}

The main analyses of well-being and diabetes-related distress used analysis of covariance (ANCOVA) and controlled for all respondent characteristics listed in Table 1 (country, residential urbanicity, age, gender, marital status).

Table 1. Sample profile

\begin{tabular}{|c|c|}
\hline Measures & $\%(N)$ or $M+S D$ \\
\hline \multicolumn{2}{|l|}{ Country } \\
\hline Australia & $9.2(317)$ \\
\hline France & $7.3(249)$ \\
\hline Germany & 8.7 (299) \\
\hline India & $8.7(299)$ \\
\hline Japan & $10.8(370)$ \\
\hline Netherlands & $10.0(344)$ \\
\hline Poland & $7.2(247)$ \\
\hline Scandinavia & $8.7(300)$ \\
\hline Spain & $7.7(264)$ \\
\hline United States & $11.2(386)$ \\
\hline United Kingdom & $10.4(357)$ \\
\hline Female & $54.2(1861)$ \\
\hline Married & $72.1(2473)$ \\
\hline \multicolumn{2}{|l|}{ Residential urbanicity } \\
\hline Rural & $22.5(772)$ \\
\hline Suburban & $20.5(705)$ \\
\hline Small urban & $18.2(626)$ \\
\hline Large urban & $38.3(1316)$ \\
\hline Age (years) & $59.28 \pm 11.84$ \\
\hline Diabetes diagnosed in last year & $6.8(232)$ \\
\hline Duration of diabetes & $11.22 \pm 9.49$ \\
\hline \multicolumn{2}{|l|}{ Medications } \\
\hline None & $12.4(425)$ \\
\hline Oral medications only & $47.7(1636)$ \\
\hline Insulin & $39.9(1371)$ \\
\hline Start insulin in last year & $4.5(155)$ \\
\hline \multicolumn{2}{|l|}{ Complications } \\
\hline None & $71.2(2444)$ \\
\hline New complications only & $6.7(229)$ \\
\hline Old complications only & $16.0(549)$ \\
\hline New and old complications & $6.1(210)$ \\
\hline Number of complications & $0.29 \pm 0.63$ \\
\hline WHO-5 well-being & $54.90 \pm 23.54$ \\
\hline Diabetes-related distress & $33.74 \pm 22.95$ \\
\hline
\end{tabular}

A single multivariate model was estimated to identify independent (additive) relationships of diabetesrelated distress and psychological well-being with each of the three state-defining criteria (duration of diabetes diagnosis, type and duration of medication use, and duration/number of complications). Several ancillary analyses were conducted using multiple regression. Two of these analyses examined whether each of the secondary criteria (duration of insulin use and number of complications) were related to the study outcomes. Two other ancillary analyses examined whether there was (a) a nonlinear relationship between number of complications and study outcomes, and (b) an interaction between number of complications and presence of a new complication.

Effect parameters (unstandardized coefficients) from multiple regression equations were used to estimate distress and well-being for each of nine states defined by combinations of the three criteria. Then these estimated levels of psychological adjustment were compared with actual levels of psychological adjustment for those classified into the states. To validate the temporal ordering of the typical diabetes states the mean duration of diabetes for people with diabetes in those states was examined; the hypothesis is a monotonic increase in duration across the nine states.

\section{Results}

Sample profile

The final sample consisted of 3432 adults with type 2 diabetes mellitus (see Table 1). Slightly over half $(54 \%)$ were female, with a median age of 59 , and most $(72 \%)$ were married. Country samples ranged from 247 to 386 , and respondents were mostly from urban locales (57\%) [23].

Most respondents (93\%) had been diagnosed with diabetes over a year (mean duration of 11 years). Only $12 \%$ did not take medication to control blood glucose, and $40 \%$ took insulin (5\% had started insulin in the last year). A third of respondents (29\%) had one or more diabetes complications, with $13 \%$ having new complications (less than 1 year duration) and 22\% had complications of longer duration. The maximum number of complications was 4, with mean of less than a third of a complication per respondent; the modest level of complications reflects the fact that respondents have a mean age of only 59 and a mean diabetes duration of only 11 years.

\section{Outcomes by state criteria}

Results are reported in Table 2. Neither well-being nor diabetes-related distress was significantly associ- 
Table 2. Least square means of well-being and diabetes-related distress for disease state markers

\begin{tabular}{|c|c|c|c|c|}
\hline \multirow[t]{2}{*}{ Disease state markers } & \multicolumn{2}{|c|}{ WHO-5 well-being } & \multicolumn{2}{|c|}{ Diabetes-related distress } \\
\hline & Mean & Std. error & Mean & Std. error \\
\hline DM Dx $<1$ year past & $53.19^{a}$ & 1.62 & $40.08^{a}$ & 1.52 \\
\hline DM Dx > 1 year past & $50.50^{a}$ & 0.68 & $38.44^{\mathrm{a}}$ & 0.64 \\
\hline No medication & $52.07^{a}, b$ & 1.39 & $33.89^{a}$ & 1.31 \\
\hline Oral medication only & $52.74^{b}$ & 1.01 & $40.39^{b}$ & 0.95 \\
\hline Insulin & $50.71^{a}$ & 1.06 & $43.49^{c}$ & 0.99 \\
\hline No complications & $58.15^{c}$ & 0.83 & $29.75^{a}$ & 0.77 \\
\hline New complications only & $48.26^{a}$ & 1.65 & $45.30^{c}$ & 1.55 \\
\hline Old complications only & $53.14^{b}$ & 1.23 & $36.56^{b}$ & 1.16 \\
\hline Old and new complications & $47.80^{\mathrm{a}}$ & 1.76 & $45.42^{c}$ & 1.66 \\
\hline
\end{tabular}

Note: Least Square means adjusted for country, age, sex, marital status, residential urbanicity, and all diabetes state markers. Means with the same superscript are not significantly different ( $p>0.05$ ); means without the same superscript are significantly different [ $p<0.005$ except insulin $v s$. oral medication only for well-being $(p=0.018)$ and new complications only vs. old complications only for well-being $(p=0.006)]$

ated with diagnosis of diabetes within the last year (given the exclusion criteria, this actually refers to diagnosis between 6 and 12 months ago). However, because the direction of association was in opposite directions for the two outcomes we conducted an additional analysis. We first reverse scored well-being so that it was an indicator of general psychological distress, then we performed a MANOVA to assess whether there was an interaction between time of diagnosis (new versus old diagnosis of diabetes) and type of distress (general versus diabetes-related). The result was a statistically significant interaction ( $p=0.008)$, with less general distress and more diabetes-related distress among those with a recent diagnosis of diabetes.

Well-being and diabetes-related distress were associated with the intensity of the glucose lowering medication regimen at different levels $(p=0.061$ and $p<0.001$, respectively). The level of well-being was lowest among those taking insulin only and highest among those taking oral medications only, with those taking no medication or both insulin and oral medications intermediate; only the two groups with the most extreme values were significantly different. Diabetesrelated distress in those taking insulin was significantly higher than the group taking only oral medication, which was significantly higher than among those not taking glucose lowering medication.

Well-being and diabetes-related distress both differed significantly $(p<0.001)$ across complication subgroups. Well-being was highest among those with no complications and significantly lower among those with only complications of more than a year's duration; those with a complication of recent onset (less than 12 months ago), with or without complications of longer duration, were significantly lower than the other groups. Diabetes-related distress followed a similar pattern - lowest among those with no complications and significantly higher among those with only complications of more than a year's duration; those with complication of recent onset (less than 12 months ago), with or without complications of longer duration, were significantly higher than the other groups.

Although our primary ANCOVA assumed only additive relationships we conducted additional ANCOVA to determine whether there were interactions (twoway or three-way) among the three main criteria. For well-being there were no significant interactions. For diabetes-related distress there was one significant ( $p=0.005$ ) interaction, between duration of diabetes and type of complications; new complications were associated with a greater elevation in distress among those with recently diagnosed diabetes.

Another ancillary analysis examined whether number of complications of over a year's duration ("old" complications) was a more powerful predictor of wellbeing and distress than merely the absence/presence of such complications. While the effect of a single old complication was much less than that of a new complication, the effect of two, or three, old complications was greater than that of a new complication (for well-being and diabetes-related distress, respectively). Another ancillary analysis demonstrated that the relationship of psychological adjustment with number of complications was linear rather than nonlinear. We also examined whether there was a significant interaction between number of old complications and the presence of a new complication; for diabetes-related distress the interaction was significant $(p=0.002)$ as the number of old complications had a much stronger impact among those with no new complication (effect 
Table 3. Duration of diabetes, well-being, and diabetes-related distress for typical states of diabetes

\begin{tabular}{|c|c|c|c|c|c|c|}
\hline DM diagnosis & Medications & Complications & $\mathbf{N}$ & DM duration & Well-being & Distress \\
\hline New & None & None & 55 & 0.9 & $59(64)$ & 29 \\
\hline Old & None & None & 314 & 7.7 & 57 & 24 \\
\hline Old & Oral only & None & 1107 & 8.8 & 58 & 30 \\
\hline Old & New insulin & None & 80 & 12.2 & 56 & 29 \\
\hline Old & Old insulin & None & 745 & 14.9 & 57 & 32 \\
\hline Old & Old insulin & New only & 105 & 17.4 & $46(48)$ & 48 \\
\hline Old & Old insulin & One old only & 183 & 18.1 & $54(52)$ & 38 \\
\hline Old & Old insulin & One old and new & 73 & 18.7 & $49(47)$ & 49 \\
\hline Old & Old insulin & Two old only & 39 & 20.5 & 40 & 48 \\
\hline
\end{tabular}

Note: Values for $\mathrm{N}$ and duration are actual values for those who fall into the groups representing each state. Values for well-being and distress are least square means obtained from regression models including diagnosis, medications, and complications. Where the actual mean for the group differs from the least square mean by more than 1 point, the actual group mean is shown in parentheses. New diagnosis, new insulin and new complications represent events taking place within one year prior to the study

parameter $=5.63, \mathrm{p}>0.001)$ than among those with a new complication (effect parameter $=2.22, \mathrm{p}=\mathrm{ns}$ ).

\section{Assessment of typical states of diabetes}

Table 3 shows that the 2701 of the 3432 participants $(79 \%)$ fell into one of the nine typical states $(80 \%$ of those with two or less complications).

Over half of those not falling into one of the typical states were people who had complications but had not yet started insulin therapy. The hypothesized temporal ordering of the typical diabetes states exactly corresponds to the actual ordering of the states as measured by the mean duration of diabetes for those in each state. Similarly, the estimated levels of wellbeing and diabetes-related distress based on the model parameters correspond closely to the actual levels, especially in the intermediate states which represent the majority of the data used to generate the estimated effect parameters.

\section{Discussion}

The findings of this study indicate that both psychological wellbeing and diabetes-related distress have similar significant independent relationships with several components of our multi-dimensional typology, including the timing (recency/latency) of events, the level of treatment intensity, and the number of complications. Moreover, the ordered set of typical states represented by a combination of these components seems to correspond closely to the actual occurrence of these states in the study sample, as indicated by the duration of diabetes for people occupying those states.

There was evidence to support the three-level categorization of treatment intensity, with each level being significantly different from another level for at least one indicator of psychological adjustment. Greater treatment intensity generally was associated with worse psychological adjustment, although those taking oral medication had slightly (i.e., not significantly) higher well-being than those not taking glucose-lowering medication.

Number of complications had the strongest relationship with psychological adjustment. Each additional complication was associated with a decrement in psychological adjustment about twice the size of the decrement associated with insulin treatment. This finding is consistent with previous research indicating that complications are associated with declines in psychological adjustment. However, recent research suggests that this relationship may be bi-directional, with depression increasing the risk of complications [24, 25] as well as the reverse [24, 26]. There is also evidence to suggest that the association between psychological adjustment and the state-defining events (change in treatment and occurrence of complications) is dependent on the timing of the events. Although time since diabetes diagnosis was not significant in the multivariate analyses based on all patients, there were substantial decrements in both indicators of psychological adjustment for those with longer time since diagnosis when the comparison was limited to those with no other risk factors (no medication and no complications). There was a similar relationship with time since initiation of insulin treatment; this was significant in multivariate analyses that controlled for occurrence of complications. Conversely, psychological adjustment was better for those whose complications were further in the past. Thus, the timing of events does not have a universal association with psychological adjustment; sometimes less recent events have a stronger association than more recent events, and sometimes vice-versa.

The temporal ordering of the typical states identified here was validated by the duration of diabetes 
associated with being in those states; the actual duration was entirely consistent with the hypothesized temporal ordering. When comparing estimated and actual levels of psychological adjustment the differences between them were mostly at the extremes and for smaller groups of people with diabetes. Since there were relatively few participants in these groups we must be cautious in making conclusions regarding these groups and the effects giving rise to differences among these groups. This is especially true for two sets of findings. For the comparison of states one and two the actual well-being scores of those with more time since diabetes diagnosis show a greater worsening than estimated scores; moreover, these states exhibit one of the few inconsistencies between the findings for wellbeing and distress, as the latter shows a substantial improvement for the same group. The second finding to view with caution is the finding regarding well-being for the onset and duration of a second complication. The estimated data necessarily shows the pattern to be the same for the initial and second complications (well-being improves as the complication recedes in time); for the actual data this pattern is replicated for the first complication, but for the second complication well-being worsens with time since the event. The pattern for actual diabetes-related distress scores is consistent with the pattern for estimated well-being scores; adjustment improves as the second complication recedes in time.

A final comment about the multivariate analyses; they demonstrate few non-additive or non-linear relationships. Number of complications did not exhibit a non-linear relationship with well-being or distress. Of the eight two-way or three-way interactions among the three main criteria for the two outcomes, only one was significant. Perhaps the most interesting departure from simple additive effects is the interaction between having a new complication and number of complications for diabetes-related distress. For patients with recent onset of a complication the number of existing complications is not associated with an increase in distress; this is confirmed by the equal levels of actual distress for those with only a new complication and those with a new complication as well as one old complication.

\section{Study strengths and limitations}

The major strength of this study is the availability of a large sample with a diverse population which allows us to compare people with diabetes with various combinations of the characteristics defining the typical states of diabetes. The fundamental limitation of this paper is the cross-sectional nature of the data used to evaluate the proposed multi-dimensional typology of diabetes states. Ideally, we would be able to follow people with diabetes over time to see whether they experience events in the order our typology hypothesizes, and prospectively observe changes in psychological adjustment associated with the transition from one state to another. Absent longitudinal data, our results are suggestive rather than definitive (although this is the first large study to comprehensively examine this issue). Another limitation is that we do not have enough people with diabetes in some states to be able to obtain reliable estimates of the differences among states; this is particularly true for assessment of the impact of newly diagnosed diabetes and recent initiation of insulin (or oral medication) therapy (fortunately, other studies provide evidence regarding the impact of these events on psychological adjustment). In addition, this study used a newly developed measure of diabetes-related distress which has not been fully validated; however, it had good reliability in assessing many of the same diabetes-related feelings (stress, worry, fear, burnout) as other measures of the same construct [14].

\section{Research implications}

Additional longitudinal studies are needed to assess the impact of the events studied here on the psychological adjustment to having diabetes. Moving beyond the issues addressed in the present research, we need to understand what factors are associated with variation in the impact of these events on the psychological adjustment of people with diabetes, e.g., outcome expectations, coping strategies, etc. Does the patient's response to events early in the progression of diabetes (e.g., acceptance of diagnoses) alter the trajectory of the progression? This knowledge would permit us to develop a more patient - centered understanding of the progression of diabetes. Research also should determine whether different complications have different consequences for psychological adjustment. Relatedly, do the benefits of treatment intensification in term of preventing complications outweigh any psychological impact of increased treatment burden? What factors in current treatment intensification, if any, contribute to psychological impact and how might new developments in diabetes therapies diminish negative impact? And while our primary analyses simply regarded diabetes-related distress and psychological well-being as independent outcomes, it is likely that they are differentially sensitive to the events studied (e.g., as shown for time since diagnosis of diabetes) and that each affects the others' course of development, a topic that warrants further research. Finally, research should examine whether the progression of states changes over time. 


\section{Clinical implications}

This study suggests that state transitions in diabetes (treatment intensification and occurrence of complications) are associated with sustained deterioration in psychological adjustment. While the psychological impact of a complication tends to wane somewhat over time, it does not disappear entirely, and it is not clear what processes produce this (partial) remission. Nevertheless, the results of this study suggest that people with diabetes experiencing these potentially traumatic events should be monitored and receive psychological treatment and support as appropriate to restore quality of life [27].

\section{Acknowledgements}

Mark Peyrot's participation in this study was supported by funding from the sponsor of the DAWN study, Novo Nordisk A/S, which also made the data available to us. The authors received no assistance in preparing this paper and are responsible for all data analyses and interpretations. We acknowledge the role of the DAWN International Advisory Board in guiding the study and their support in the dissemination of study findings.

The preliminary data from the present study were partially presented at the 15th Scientific Meeting of the PSAD (PsychoSocial Aspects of Diabetes) Study Group [23].

\section{REFERENCES}

1. Weir GC, Bonner-Weir S. Five stages of evolving beta-cell dysfunction during progression to diabetes. Diabetes. 2004; 53 Suppl 3: S16-S21, doi: 10.2337/diabetes.53.suppl_3.s16, indexed in Pubmed: 15561905

2. Alberti K, Zimmet PZ. Definition, diagnosis and classification of diabetes mellitus and its complications. Part 1: diagnosis and classification of diabetes mellitus. Provisional report of a WHO Consultation. Diabetic Medicine. 1998; 15(7): 539-553, doi: 10.1002/ (sici)1096-9136(199807)15:7<539::aid-dia668>3.0.co;2-s.

3. Fonseca VA. Defining and characterizing the progression of type 2 diabetes. Diabetes Care. 2009; 32 Suppl 2: S151-S156, doi: 10.2337/dc09-S301, indexed in Pubmed: 19875543.

4. Seino Y, Nanjo K, Tajima N, et al. Committee of the Japan Diabetes Society on the Diagnostic Criteria of Diabetes Mellitus. Report of the committee on the classification and diagnostic criteria of diabetes mellitus. J Diabetes Investig. 2010; 1(5): 212-228, doi: 10.1111/j.2040-1124.2010.00074.x, indexed in Pubmed: 24843435.

5. Gonnella JS (ed.). Disease staging: clinical and coded criteria. Version 5.27. Thomson Reuters, Ann Arbor, Michigan 2010.

6. Hamburg BA, Innoff GE. Coping with predictable crises of diabetes. Diabetes Care. 1983; 6: 409-416.

7. Mezuk B, Eaton WW, Albrecht S, et al. Depression and type 2 diabetes over the lifespan: a meta-analysis. Diabetes Care. 2008; 31(12): 2383-2390, doi: 10.2337/dc08-0985, indexed in Pubmed: 19033418.

8. Palinkas LA, Barrett-Connor E, Wingard DL. Type 2 Diabetes and Depressive Symptoms in Older Adults: a Population-based Study.
Diabetic Medicine. 1991; 8(6): 532-539, doi: 10.1111/j.14645491.1991.tb01646.x.

9. Noh JH, Park JK, Lee HJ, et al. Depressive symptoms of type 2 diabetics treated with insulin compared to diabetics taking oral anti-diabetic drugs: a Korean study. Diabetes Res Clin Pract. 2005; 69(3): 243-248, doi: 10.1016/j.diabres.2004.10.009, indexed in Pubmed: 16046024.

10. Li C, Ford ES, Strine TW, et al. Prevalence of depression among U.S. adults with diabetes: findings from the 2006 behavioral risk factor surveillance system. Diabetes Care. 2008; 31(1): 105-107, doi: 10.2337/dc07-1154, indexed in Pubmed: 17934145.

11. Hendra TJ, Taylor CD. A randomised trial of insulin on well-being and carer strain in elderly type 2 diabetic subjects. J Diabetes Complications. 2004; 18(3): 148-154, doi: 10.1016/j.jdiacomp.2003.11.001, indexed in Pubmed: 15145325.

12. Reza M, Taylor CD, Towse K, et al. Insulin improves well-being for selected elderly type 2 diabetic subjects. Diabetes Res Clin Pract. 2002; 55(3): 201-207, indexed in Pubmed: 11850096.

13. Peyrot M, Rubin RR. Levels and risks of depression and anxiety symptomatology among diabetic adults. Diabetes Care. 1997; 20(4): 585-590, doi: 10.2337/diacare.20.4.585, indexed in Pubmed: 9096984.

14. Fisher L, Mullan JT, Skaff MM, et al. Predicting diabetes distress in patients with Type 2 diabetes: a longitudinal study. Diabet Med. 2009; 26(6): 622-627, doi: 10.1111/j.1464-5491.2009.02730.x, indexed in Pubmed: 19538238.

15. Semenkovich K, Brown ME, Svrakic DM, et al. Depression in type 2 diabetes mellitus: prevalence, impact, and treatment. Drugs. 2015; 75(6): 577-587, doi: 10.1007/s40265-015-0347-4, indexed in Pubmed: 25851098.

16. Perrin NE, Davies MJ, Robertson $N$, et al. The prevalence of diabetes-specific emotional distress in people with Type 2 diabetes: a systematic review and meta-analysis. Diabet Med. 2017; 34(11): 1508-1520, doi: 10.1111/dme.13448, indexed in Pubmed: 28799294.

17. De Berardis G, Pellegrini F, Franciosi M, et al. QuED (Quality of Care and Outcomes in Type 2 Diabetes) Study Group. Longitudinal assessment of quality of life in patients with type 2 diabetes and self-reported erectile dysfunction. Diabetes Care. 2005; 28(11): 2637-2643, doi: 10.2337/diacare.28.11.2637, indexed in Pubmed: 16249532.

18. Bernbaum M, Albert SG, Duckro PN. Psychosocial profiles in patients with visual impairment due to diabetic retinopathy. Diabetes Care. 1988; 11(7): 551-557, doi: 10.2337/diacare.11.7.551, indexed in Pubmed: 3203572.

19. Peyrot M, Rubin RR, Lauritzen T, et al. Patient and provider perceptions of care for diabetes: results of the cross-national DAWN Study. Diabetologia. 2006; 49(2): 279-288, doi: 10.1007/s00125005-0048-8, indexed in Pubmed: 16397792.

20. Peyrot M, Rubin RR, Lauritzen T, et al. Psychosocial problems and barriers to improved diabetes management: results of the CrossNational Diabetes Attitudes, Wishes and Needs (DAWN) Study. Diabet Med. 2005; 22(10): 1379-1385, doi: 10.1111/j.14645491.2005.01644.x, indexed in Pubmed: 16176200.

21. Bonsignore $M$, Barkow $K$, Jessen $F$, et al. Validity of the five-item WHO Well-Being Index (WHO-5) in an elderly population. Eur Arch Psychiatry Clin Neurosci. 2001; 251 Suppl 2: II27-II31, indexed in Pubmed: 11824831.

22. Topp CW, Østergaard SD, Søndergaard S, et al. The WHO-5 WellBeing Index: a systematic review of the literature. Psychother Psychosom. 2015; 84(3): 167-176, doi: 10.1159/000376585, indexed in Pubmed: 25831962.

23. Peyrot M. Psychological well-being and diabetes-related distress across stages of type 2 diabetes in the multi-national Diabetes Attitudes, Wishes and Needs (DAWN) Study. The 15th Scientific Meeting of the PSAD Study Group, PsychoSocial Aspects of Diabetes Study Group, Cambridge, UK, 16-18 April 2010. Abstract booklet. 29 . 
24. Lin EHB, Rutter CM, Katon W, et al. Depression and advanced complications of diabetes: a prospective cohort study. Diabetes Care. 2010; 33(2): 264-269, doi: 10.2337/dc09-1068, indexed in Pubmed: 19933989.

25. Nefs G, Pop VJ, Denollet J, et al. Depressive Symptom Clusters Differentially Predict Cardiovascular Hospitalization in People With Type 2 Diabetes. Psychosomatics. 2015; 56(6): 662-673, doi: 10.1016/j.psym.2015.06.006, indexed in Pubmed: 26481961.
26. Novak M, Mucsi I, Rhee CM, et al. Increased Risk of Incident Chronic Kidney Disease, Cardiovascular Disease, and Mortality in Patients With Diabetes With Comorbid Depression. Diabetes Care. 2016; 39(11): 1940-1947, doi: 10.2337/dc16-0048, indexed in Pubmed: 27311494.

27. Young-Hyman D, de Groot M, Hill-Briggs F, et al. Psychosocial Care for People With Diabetes: A Position Statement of the American Diabetes Association. Diabetes Care. 2016; 39(12): 2126-2140, doi: 10.2337/dc16-2053, indexed in Pubmed: 27879358. 